\title{
A variation in NOS1AP gene is associated with repaglinide efficacy on insulin resistance in type 2 diabetes of Chinese
}

\author{
Wen QIN", Rong ZHANG\#, Cheng HU, Cong-rong WANG, Jing-yi LU, Wei-hui YU, Yu-qian BAO, Kun-san XIANG, International \\ Type 2 Diabetes 1q Consortium, Wei-ping JIA* \\ Shanghai Diabetes Institute, Department of Endocrinology and Metabolism, Shanghai Jiao Tong University Affiliated Sixth People's \\ Hospital, Shanghai 200233, China
}

\begin{abstract}
Aim: To investigate a potential association between SNP rs10494366 in the neural nitric oxide synthase adaptor protein (NOS1AP) and efficacy of repaglinide (an insulin secretagogue) in newly diagnosed Shanghai Chinese type 2 diabetes patients.

Methods: A total of 104 newly diagnosed type 2 diabetes patients (69 men, 35 women) were recruited and treated with repaglinide for 24 weeks. Anthropometric measurements, clinical laboratory tests were obtained at baseline and after 24-week treatment. Genotyping was performed by sequencing.

Results: The baseline value of BMI, HOMA-IR, HOMA-B, and fasting insulin level were significantly different between GG, GT, and TT genotypes $(P=0.024,0.030,0.005$, and 0.007 , respectively). Carriers of TT genotype were in significant insulin resistance at baseline. After 24-week repaglinide monotherapy, the $\Delta$ value of fasting insulin $(P=0.019)$ and HOMA-IR $(P=0.011)$ were significantly different. TT carriers had the least insulin resistance after treatment. The mixed model analysis showed that the variation had an interaction effect with repaglinide treatment only on HOMA-IR $(P=0.013)$.

Conclusion: A common variant in rs10494366 is associated with repaglinide monotherapy efficacy on insulin resistance in newly diagnosed Shanghai Chinese type 2 diabetes patients.
\end{abstract}

Keywords: pharmacogenetics; repaglinide; single nucleotide polymorphisms; insulin resistance; nitric oxide synthase 1 (neuronal) adaptor protein; NOS1AP

Acta Pharmacologica Sinica (2010) 31: 450-454; doi: 10.1038/aps.2010.25; published online 22 Mar 2010

\section{Introduction}

The oral anti-diabetic drugs, such as sulfonylureas, meglitinides, dimethylbiguanide, and inhibitor of alpha glucosidase are commonly used for the treatment of type 2 diebetes mellitus. The clinical response to these oral agents varies among individuals, and genetic predisposition within nitric oxide synthase 1 adaptor protein (NOS1AP) has been suggested ${ }^{[1]}$.

Neuronal nitric oxide synthase (nNOS) is a member of the nitric oxide synthase (NOSs) family that also includes endothelial nitric oxide synthase (eNOS) and inducible nitric oxide synthase (iNOS) ${ }^{[2]}$. NOSs null mice all demonstrated insulin resistance ${ }^{[3-7]}$. Insulin signal pathway [phosphatidylinositol-3 kinase (PI3K)-protein kinase B (AKT)-NOS axis] has been implicated in brain insulin resistance ${ }^{[5]}$. NO derived

\footnotetext{
\# These authors contribute equally to this article.

* To whom correspondence should be addressed.

E-mail wpjia@sjtu.edu.cn

Received 2009-06-04 Accepted 2010-02-05
}

from nNOS plays a role in facilitating glucose uptake and insulin sensitivity ${ }^{[3,6]}$. Moreover, it inhibits central sympathetic outflow and counteracts sympathetic vasoconstriction ${ }^{[7]}$. Besides, nNOS in pancreatic beta cell has been shown to play a role in insulin release through induction of intracellular $\mathrm{Ca}^{2+[8]}$.

Nitric oxide synthase 1 adaptor protein (NOS1AP), also known as CAPON, is localized primarily in the mitochondria and cytoplasm; and can inhibit nNOS function via binding to nNOS PDZ motif ${ }^{[9]}$. Currently, variants in NOS1AP were found to be associated with type 2 diabetes mellitus ${ }^{[10,11]}$. It is unknown whether or not binding of NOS1AP to nNOS will affect the insulin signal pathway. Nor is it clear if the common variation in NOS1AP influences insulin secretion and/ or insulin resistance. In this study, we determined the variant and the efficacy of repaglinide (one of the oral anti-diabetic meglitinides that stimulates insulin secretion by blocking ATPsensitive $\mathrm{K}^{+}$channels to modulate intracellular calcium in beta cells $^{[12]}$ ) in newly diagnosed Shanghai Chinese type 2 diabetes patients who had been treated with repaglinide for 24 weeks. 


\section{Materials and methods}

\section{Patients and study design}

Newly diagnosed type 2 diabetes patients, diagnosed on the basis of the World Health Organization criteria ${ }^{[13]}$, were recruited from outpatient clinics of ten hospitals in Shanghai. The patients were between 30 and 70 years old with entry glycated hemoglobin value $>6.5 \%$, and did no antihyperglycemic therapies prior to the study. Exclusion criteria include diabetic ketoacidosis, nonketotic hyperosmolar coma, chronic diabetic complication with fasting plasma glucose (FPG) >13 $\mathrm{mmol} / \mathrm{L}(234 \mathrm{mg} / \mathrm{dL}$ ) and/or $2 \mathrm{~h}$ postprandial plasma glucose (2-h PG) >18 mmol/L (324 mg/dL) (details were described previously) ${ }^{[13]}$. We recruited 104 patients (69 men, 35 women) and treated them with repaglinide for 24 weeks. Repaglinide was administrated initially in a mealtime dosage of $0.5 \mathrm{mg}$ and later increased stepwise to 1, 1.5, and $2 \mathrm{mg}$ for patients who failed to achieve glycemic targets of fasting plasma glucose $<7$ $\mathrm{mmol} / \mathrm{L}(126 \mathrm{mg} / \mathrm{dL})$ and/or $2 \mathrm{~h}$ plasma glucose $<11 \mathrm{mmol} / \mathrm{L}$ (200 mg/dL). Experimental protocols used in this study were approved by the institutional review board of Shanghai Jiao Tong University Affiliated Sixth People's Hospital. Subjects were given informed written consent.

\section{Anthropometric measurements}

The general anthropometric parameters considered for the study were height $(\mathrm{m})$, weight $(\mathrm{kg})$, waist and hip circumferences $(\mathrm{cm})$. All patients were measured at baseline of the study and 24 weeks after the initiation of the repaglinide therapy. The body mass index (BMI) and waist-hip ratio were calculated as weight/height ${ }^{2}$ and waist/hip, respectively.

\section{Clinical laboratory tests}

Overnight, fasting and 2-h blood samples [following a $75 \mathrm{~g}$ oral glucose tolerance test (OGTT)] were collected. Glycated hemoglobin values were determined by high-performance liquid chromatography with a Bio-Rad Variant II hemoglobin testing system (Bio-Rad Laboratories, Hercules, CA, USA). Insulin resistance and beta cell function were assessed by homeostasis model assessment (HOMA). Homeostasis model assess the insulin resistance (HOMA-IR) is calculated using the formula: $=$ fasting insulin concentration $($ in $\mathrm{mU} / \mathrm{L}) \times$ fasting plasma glucose concentration (in mmol/L)/22.5, and homeostasis model assessment beta cell function (HOMA-B) is calculated using the formula: $=20 \times$ fasting insulin concentration/ (fasting plasma glucose concentration-3.5) ${ }^{[14]}$. Intravenous arginine stimulation tests were performed under fasting conditions and the acute insulin response to arginine (AIR, in $\mathrm{mU} / \mathrm{L}$ ) was calculated as the mean insulin value of 2,4 , and 6 min samples minus fasting insulin concentration to evaluate the potential function of beta cells, and acute insulin secretion after adjusted for plasma glucose was calculated as well ${ }^{[14]}$.

\section{Genotyping}

Genomic DNA was extracted from peripheral blood leucocytes in whole blood samples. SNP was identified by PCR amplification followed by DNA sequencing. PCR amplification was carried out on the GeneAmp PCR system 9700 (Applied Biosystems, Foster City, CA, USA), and DNA sequencing was carried out using 3100 Genetic Analyzer (Applied Biosystem, Foster City, CA, USA).

\section{Statistical analysis}

The allele frequencies determined by gene calculating and the Hardy-Weinberg equilibrium tests were performed ${ }^{[15]}$. Parameters (expressed as mean \pm SEM) presented in Table1 was determined for normal distribution by the Shapiro-Wilk test. Differences between parameters with normal distribution were tested using the multiple linear regression (adjusted for sex, age, BMI, genotype, and hospitals), and differences between parameters with abnormal distribution were tested by the Kruskal-Wallis Test. The parameters tested include (i) baseline value, (ii) value at 24 weeks after repaglinide therapy, and (iii) $\Delta$ value between (i) and (ii). All of these analyses were performed using SAS for Windows (version 6.12; SAS Institute, Cary, NC). A two-tailed $P$ value $<0.05$ was considered statistically significant. Mixed effect model analysis was performed using SPSS for Windows (version 13.0; SPSS Inc, Chicago, IL, USA) to test interaction effect between genotype and repaglinide treatment. Parameters of each individual at pre and post 24-week repaglinide treatment were considered as repeated measure factor. Compound symmetry was chosen as covariance structure. Covariates were sex, age, BMI, and centers.

\section{Results}

Of total 104 patients recruited, 100 (66 men, 34 women) completed the 24-week study. Two patients were excluded because of glycated hemoglobin $\geq 8 \%$ at two consecutive times, and two were dropped out. The GG, GT, TT genotype distribution among the patients was 40,50, and 10, respectively. The alleles of the G/T variant in rs10494366 were 65 and 35 . The minor allele frequency was 0.350 (T allele) and genotype distribution was in agreement with the Hardy-Weinberg equilibrium $(P=0.323)^{[15]}$. The subjects were divided into three groups according to NOS1AP genotype.

The baseline value of BMI, HOMA-IR, HOMA-B, and fasting insulin level among GG, GT, and TT genotypes were significantly different $(P=0.024,0.030,0.005$, and 0.007 , respectively) (Table 1). The values of these four clinic parameters all increased from GG, GT to TT genotype.

The $\Delta$ values of parameters at pre and post treatment between different genotypes were determined by multiple linear regression or Kruskal-Wallis Test when appropriate; the results showed that the $\Delta$ values of HOMA-IR $(P=0.011)$ and fasting insulin $(P=0.019)$ were statistically significant between the three groups (Table 1). However, the baseline values of HOMA-IR $(P=0.030)$ and fasting insulin $(P=0.007)$ were significant different as well. To determine whether the fasting insulin level observed among different groups was attributable to baseline influence or due to an interaction effect between the variant of rs10494366 and repaglinide treatment, we performed mixed effect model test. Results of the mixed effect 
Table 1. Association between SNP rs10494366 in NOS1AP and clinical traits. Data are mean \pm SEM.

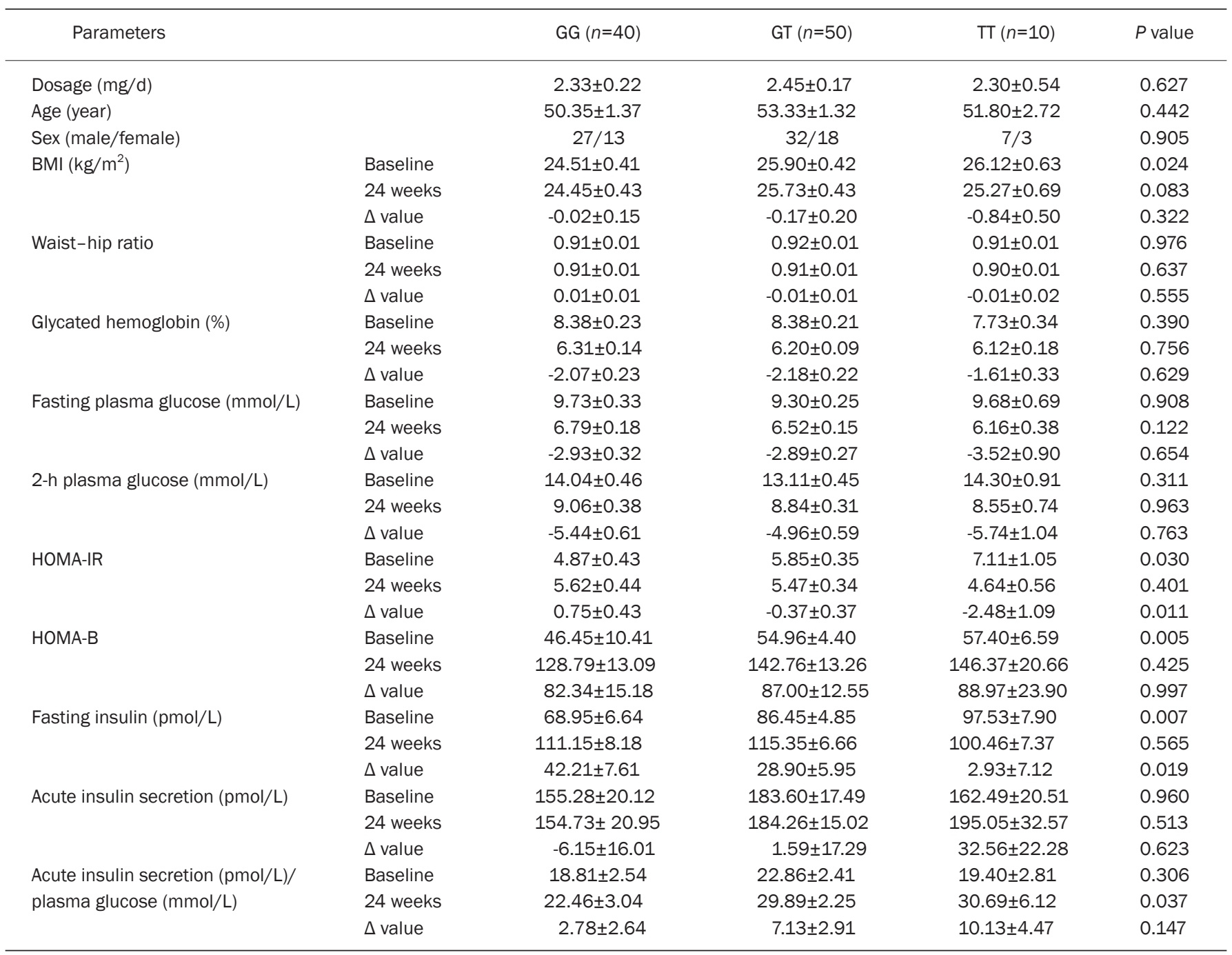

BMI, body mass index; HOMA-IR, homeostasis model assessment of insulin resistance; HOMA-B, homeostasis model assessment of beta cell function; $\Delta$ value, which equals the value at 24 weeks minus the value at baseline.

model revealed that repaglinide treatment had effect on BMI, glycated hemoglobin, and fasting plasma glucose, 2-h plasma glucose, HOMA-B, fasting insulin $(P=0.039,<0.001,<0.001$, $<0.001,<0.001$, and $<0.001$, respectively). However, the variant of rs10494366 and repaglinide treatment had an interaction effect only in HOMA-IR $(P=0.013)$ (Table 2). Therefore, under the treatment of repaglinide, patients in different genotypes respond differently to the drug in HOMA-IR.

Other parameters, such as glycated hemoglobin, fasting plasma glucose, 2-h plasma glucose, BMI, waist-hip ratio, HOMA-B, fasting insulin, acute insulin secretion (AIR) or acute insulin secretion after adjusted for plasma glucose, did not show genotype and treatment interaction effect (Table 2).

\section{Discussion}

The present preliminary study with newly identified type 2 diabetes patients of Shanghai Chinese showed that the variant of rs10494366 in NOS1AP has an association with repaglinide treatment in HOMA-IR. At beseline, patients in TT genotype developed the highest insulin resistance (HOMA-IR=7.11), compared with GT (5.85) and GG (4.87) carriers $(P=0.03)$ (Table 1). This is probably due to paralleled obese status: the BMI of TT carriers was the highest, followed by GT and GG genotypes $(P=0.024)$ (Table 1$)$. TT carriers who developed higher insulin resistance (because of obesity) would increase more insulin secretion (fasting insulin: TT 97.53; GT 86.45; GG 68.95) to maintain glucose level as similar as other genotypes (for instance, fasting plasma glucose, TT 9.68; GT 9.30; GG 9.73) (Table 1). Therefore, TT carriers seem to have higher risk than other genotypes to develop type 2 diabetes due to their highest insulin resistance.

An important mechanism of repaglinide in treating patients is to improve first phase insulin secretion (first-phase insulin secretion occurs within first 5-10 min following glucose, 
Table 2. Mixed effect model analysis of 24-week repaglinide treatment effect, genotypes effect and their interaction effect.

\begin{tabular}{|c|c|c|c|}
\hline Parameters & $\begin{array}{c}\text { Repaglinide } \\
\text { treatment effect } \\
\quad(P \text { value })\end{array}$ & $\begin{array}{c}\text { Genotypes effect } \\
(P \text { value })\end{array}$ & $\begin{array}{c}\text { Genotypes and treatment } \\
\text { interaction effect } \\
(P \text { value })\end{array}$ \\
\hline $\mathrm{BMI}\left(\mathrm{kg} / \mathrm{m}^{2}\right)$ & 0.039 & 0.064 & 0.198 \\
\hline Waist-hip ratio & 0.907 & 0.804 & 0.527 \\
\hline Glycated hemoglobin (\%) & $<0.001$ & 0.427 & 0.585 \\
\hline HOMA-IR & 0.056 & 0.824 & 0.013 \\
\hline HOMA-B & $<0.001$ & 0.881 & 0.806 \\
\hline Fasting insulin (pmol/L) & $<0.001$ & 0.812 & 0.103 \\
\hline Acute insulin secretion(pmol/L) & 0.270 & 0.875 & 0.395 \\
\hline Acute insulin secretion (pmol/L)/Plasma glucose (mmol/L) & 0.769 & 0.032 & 0.738 \\
\hline
\end{tabular}

arginine or drug stimulation), which can lower 2-h plasma glucose, inhibit hepatic glucose output, reduce insulin resistance $^{[16,17]}$. Although there is no significant difference in acute insulin secretion between groups after repaglinide treatment for 24 weeks, the trend should be noticed that without drug dose difference, acute insulin secretion effect in TT carriers increased more from baseline (162) to 195; while GG or GT had no obvious increase (for GG, baseline is 155 and after 24 weeks is 154; likewise, for GT, baseline is 183 and after 24 weeks is 184) (Table 1). HOMA-IR in TT carriers reduced from baseline (7.1) to 4.6, while, HOMA-IR in GT genotype reduced from baseline (5.8) to 5.5. For GG carriers, HOMA-IR increased after treatment (from 4.8 to 5.6) (Table 1). One of the possibilities may be that GG carriers lack the increment of first phase insulin secretion to reduce HOMA-IR obviously as TT carriers did. Apparently for the GG genotypes to achieve the same glucose lowering, more fasting insulin are required than the TT carriers $(P=0.019)$ (Table 1$)$. Another possibility may be that GG genotype had more severe insulin resistance during repaglinide treatment. Thus, HOMA-IR in GG genotype increased after treatment. Results of the mixed effect model revealed that the variant of rs10494366 and repaglinide treatment had an interaction effect in HOMA-IR $(P=0.013)$ (Table 2). Despite of more serious insulin resistance at baseline, TT carriers responded more actively to the treatment of repaglinide in decreasing HOMA-IR than other genotypes.

The underlying mechanism responsible for the observed interaction effect between variant of rs10494366 and HOMA-IR upon repaglinide treatment is unclear. Several possibilities for the interaction effect may be considered. First, although variant of rs10494366 is located within the intron 1 of NOS1AP thus it is unlikely to be a causal SNP, this polymorphism could potentially be a modulation site acted upon by micro RNA. Intronic micro RNA recognizing sequences are found in many genes including eNOS ${ }^{[18]}$. It will be of interest to determine whether or not the NOS1AP transcript could be regulated by this mechanism. Alternatively, variant of rs10494366 may represent a genetic marker that is in linkage-disequilibrium (LD) with an unidentified causal genetic variant.

The second possibility may relate to insulin secretion: nNOS in pancreatic beta cell has been shown to play a role in insulin release through induction of intracellular $\mathrm{Ca}^{2+[8]}$. When using the same dosage of repaglinide, TT carriers respond more actively to increase acute insulin secretion than other genotypes, which will help to reduce HOMA-IR to a certain extent. However, different genotypes may also have the interaction effect with repaglinide treatment in peripheral insulin resistance. In the brain, increase in insulin upon treatment of repaglinide could activate the PI3K-AKT-NOS pathway and enhance NOS phosphorylation ${ }^{[5]}$. It was shown that administration of insulin into rat brain nucleus tractus solitarll activated PI3K and nNOS phosphorylation, and the resulting p-nNOS translocated from out synaptic space to inside synaptic space ${ }^{[5]}$. The cytosolic NOS1AP can bind $\mathrm{nNOS}$ and inhibit its NO synthesis function ${ }^{[9]}$. The brain NO attenuates sympathetic nerve activity to facilitate glucose disposal, and sympathetic hyperactivity is associated with insulin resistance ${ }^{[19,20]}$. Alternatively, the nNOS derived NO may also modulate insulin sensitivity in skeletal muscle by enhancing glucose uptake and improve mitochondria function ${ }^{[6,21]}$. Whether the variant in NOS1AP exerts inhibitory effect on $\mathrm{nNOS}$ function confers insulin resistance or insulin secretion needs to be determined experimentally. Multiple traits were analyzed in our study and the significant would disappear if we used a Bonferroni correction to adjust for the multiple comparison, thus we cannot exclude the possibility that our finding is false positive. However, as all the traits we analyzed are highly related, eg, glucose, insulin levels and HOMA-IR, HOMA-B, the impact of multiple comparison will be limited in the current study.

In summary, the present pharmacogenomics studies have demonstrated that although the insulin secretagogue repaglinide treatment could achieve glucose lowering to similar extent among all type 2 diabetes patients tested, the variant of rs10494366 in NOS1AP might have an influence on insulin response in type 2 diabetes: T carriers had more serious insulin resistance before treatment, but responded more actively 
to repaglinide in decreasing HOMA-IR than other genotype carriers. These data underscore the importance of taking into consideration of variant of rs10494366 in NOS1AP in the tailored anti-diabetic drug treatment.

\section{Acknowledgements}

This work was funded by the National Natural Science Foundation of China (30630061), National 973 Program (2006CB503901), National 863 Program (2006AA02A409), key project of Science and Technology Commission of Shanghai Municipality (01ZD002), major program of Shanghai Municipality for Basic Research (08dj1400601), Shanghai Key Laboratory of Diabetes Mellitus (08DZ2230220), National Institutes of Health/National Institute of Diabetes and Digestive and Kidney Diseases (U01-DK58026 and R01-DK073490).

We appreciate all the patients participating in this research. We are grateful to the doctors and nurses who participated in this study. We appreciate Professor Ze-min YAO (University of Ottawa) a critical reading of the manuscript.

\section{Author contribution}

Wen QIN and Rong ZHANG performed research; Wen QIN and Cheng HU analyzed data; Cheng HU, Yu-qian BAO, Kun-san XIANG, Wei-ping JIA designed research; Cong-rong WANG, Jing-yi LU and Wei-hui YU contributed new reagents or analytic tools; Wen QIN wrote the paper, Cheng HU and Wei-ping JIA critically revised the manuscript.

\section{References}

1 Becker ML, Aarnoudse AJ, Newton-Cheh C, Hofman A, Witteman JC, Uitterlinden AG, et al. Common variation in the NOS1AP gene is associated with reduced glucose-lowering effect and with increased mortality in users of sulfonylurea. Pharmacogenet Genomics 2008; 18: 591-7.

2 Bredt DS. Endogenous nitric oxide synthesis: biological functions and pathophysiology. Free Radic Res 1999; 31: 577-96.

3 Nisoli E, Clementi E, Paolucci C, Cozzi V, Tonello C, Sciorati C, et al. Mitochondrial biogenesis in mammals: the role of endogenous nitric oxide. Science 2003; 299: 896-9.

4 Duplain H, Burcelin R, Sartori C, Cook S, Egli M, Lepori M, et al. Insulin resistance, hyperlipidemia, and hypertension in mice lacking endothelial nitric oxide synthase. Circulation 2001; 104: 342-5.

5 Chiang HT, Cheng WH, Lu PJ, Huang HN, Lo WC, Tseng YC, et al. Neuronal nitric oxide synthase activation is involved in insulinmediated cardiovascular effects in the nucleus tractus solitarii of rats. Neuroscience 2009; 159: 727-34.

6 Scherrer U, Randin D, Vollenweider P, Vollenweider L, Nicod P. Nitric oxide release accounts for insulin's vascular effects in humans. J Clin
Invest 1994; 94: 2511-5.

7 Turini P, Thalmann S, Jayet PY, Cook S, Mathieu C, Burcelin R, et al. Insulin resistance in mice lacking neuronal nitric oxide synthase is related to an alpha-adrenergic mechanism. Swiss Med Wkly 2007; 137: 700-4.

8 Lajoix AD, Reggio H, Chardes T, Peraldi-Roux S, Tribillac F, Roye $\mathrm{M}$, et al. A neuronal isoform of nitric oxide synthase expressed in pancreatic beta-cells controls insulin secretion. Diabetes 2001; 50 : 1311-23.

9 Jaffrey SR, Snowman AM, Eliasson MJ, Cohen NA, Snyder SH. CAPON: a protein associated with neuronal nitric oxide synthase that regulates its interactions with PSD95. Neuron 1998; 20: 115-24.

10 Chu AY, Coresh J, Arking DE, Pankow JS, Tomaselli GF, Chakravarti A, et al. NOS1AP variant associated with incidence of type 2 diabetes in calcium channel blocker users in the Atherosclerosis Risk in Communities (ARIC) study. Diabetologia 2010; 53: 510-6.

$11 \mathrm{Hu}$ C, Wang C, Zhang R, Ng MC, Bao Y, So WY, et al. Association of genetic variants of NOS1AP with type 2 diabetes in a Chinese population. Diabetologia 2010; 53: 290-8.

12 Scheen AJ. Drug-drug and food-drug pharmacokinetic interactions with new insulinotropic agents repaglinide and nateglinide. Clin Pharmacokinet 2007; 46: 93-108.

13 He YY, Zhang R, Shao XY, Hu C, Wang CR, Lu JX, et al. Association of KCNJ11 and ABCC8 genetic polymorphisms with response to repaglinide in Chinese diabetic patients. Acta Pharmacol Sin 2008; 29: 983-9.

14 Hu C, Wang C, Zhang R, Ma X, Wang J, Lu J, et al. Variations in KCNQ1 are associated with type 2 diabetes and beta cell function in a Chinese population. Diabetologia 2009; 52: 1322-5.

15 Wigginton JE, Cutler DJ, Abecasis GR. A note on exact tests of HardyWeinberg equilibrium. Am J Hum Genet 2005; 76: 887-93.

16 Hollingdal M, Sturis J, Gall MA, Damsbo P, Pincus S, Veldhuis JD, et al. Repaglinide treatment amplifies first-phase insulin secretion and highfrequency pulsatile insulin release in Type 2 diabetes. Diabet Med 2005; 22: 1408-13.

17 Del Prato S, Tiengo A. The importance of first-phase insulin secretion: implications for the therapy of type 2 diabetes mellitus. Diabetes Metab Res Rev 2001; 17: 164-74.

18 Zhang MX, Ou H, Shen YH, Wang J, Coselli J, Wang XL. Regulation of endothelial nitric oxide synthase by small RNA. Proc Natl Acad Sci USA 2005; 102: 16967-72.

19 Sartori C, Scherrer U. Insulin, nitric oxide and the sympathetic nervous system: at the crossroads of metabolic and cardiovascular regulation. J Hypertens 1999; 17: 1517-25.

20 Scherrer U, Sartori C. Insulin as a vascular and sympathoexcitatory hormone: implications for blood pressure regulation, insulin sensitivity, and cardiovascular morbidity. Circulation 1997; 96: 4104-13.

21 Steinberg HO, Brechtel G, Johnson A, Fineberg N, Baron AD. Insulinmediated skeletal muscle vasodilation is nitric oxide dependent. A novel action of insulin to increase nitric oxide release. J Clin Invest 1994; 94: 1172-9. 